\title{
Lung Ultrasound: The Emerging Role of Respiratory Therapists
}

\author{
Manjush Karthika, Duane Wong, Suresh G Nair, Lalitha V Pillai, and Chris Sara Mathew
}

\author{
Introduction \\ Technical Aspects of Lung Ultrasound \\ Clinical Applications of Lung Ultrasound \\ Lung Ultrasound by Non-Physicians \\ The Emerging Role of Respiratory Therapists \\ Recognition of Pneumothorax \\ Weaning From Mechanical Ventilation \\ Airway Management \\ Competency and Training in Lung Ultrasound \\ Conclusion \\ Directions of Future Research
}

\begin{abstract}
Lung ultrasound is a point-of-care imaging tool that is routinely used in acute care medicine. Traditionally, radiology physicians were the primary practitioners of diagnostic ultrasound, but with the recognition of its importance in intensive care medicine, critical care physicians have also adopted this practice. Within the intensive care unit inter-professional team is the respiratory therapist, who participates actively in the care of ventilated patients. Their scope of responsibility is expanding with newer technologies being brought into clinical use on a regular basis. This review focuses on the scope and benefits of ultrasound training within respiratory care-related areas. Key words: lung ultrasound; respiratory therapists; intensive care units. [Respir Care 2019;64(2):217-229.

(c) 2019 Daedalus Enterprises]
\end{abstract}

\section{Introduction}

The portable chest radiograph is a routine diagnostic tool used in the ICU setting to assess patient lung function.

\footnotetext{
Dr Karthika and Mr Wong are affiliated with the Department of Respiratory Services, Sidra Medicine, Doha, Qatar. Dr Nair is affiliated with the Department of Anesthesia and Critical Care Medicine, Aster Medcity Hospital, Kochi, Kerala, India. Dr Pillai is affiliated with the Department of Critical Care Medicine, Aundh Institute of Medical Sciences, Pune, Maharashtra, India. Ms Mathew is affiliated with the College of Health Sciences, Srinivas University, Mangalore, Karnataka, India.
}

The authors have disclosed no conflicts of interest.

Correspondence: Dr Manjush Karthika, Department of Respiratory Services, Sidra Medicine, Doha, Qatar. Email: manjushnair@hotmail.com.

DOI: $10.4187 /$ respcare.06179
The routine use of bedside chest radiography is supported by longstanding data, ${ }^{1}$ but there are studies that question its diagnostic impact and clinical efficacy. ${ }^{2-4}$ Portable chest radiography has gradually appeared to be less useful, as noted in a large meta-analysis of randomized, controlled trials and observational studies. ${ }^{5}$

Computed tomography remains the accepted standard for all diagnostic and therapeutic procedures that require evaluation of lung function, whether for diagnostic purposes in pneumothorax, pneumonia, or pleural effusion, or for therapeutic purposes such as drainage of loculated or large effusions and for insertion of intercostal or pig-tail catheters. However, transporting a critically ill ICU patient with all of the accompanying monitoring equipment and emergency preparedness may not always be a practical option. ${ }^{6}$

The advancement of lung ultrasound (LUS) in recent years with better quality and spatial resolution has resulted in greater diagnostic accuracy. ${ }^{7}$ Some of the advantages of 
LUS over chest radiograph and computed tomography include availability, portability, absence of radiation, realtime imaging, documentation, and reproducibility of findings. Over the last 20 years, LUS has become a prominent diagnostic tool for assessment and decision-making in care of the ventilated patient.

\section{Technical Aspects of Lung Ultrasound}

Ultrasound waves have frequencies higher than the upper limit of the human audible range, which is $2-20 \mathrm{KHz}$. Diagnostic ultrasound in medical science typically uses frequencies that range between 2 and 20MHz. Ultrasound waves are generated with piezoelectric crystals located in the head of the transducer or probe of the ultrasound machine. ${ }^{8}$ When electrical energy is transmitted through the piezoelectric crystals in the transducer of the ultrasound machine, it deforms the crystals and ultrasound waves are generated. These ultrasound waves are then transmitted, attenuated, absorbed, reflected, refracted, and diffracted by the tissues or adjacent medium. ${ }^{9}$ Although nearly all of the energy is reflected back, the difference in the acoustic impedance of various tissues changes the ultrasound signal strength. This provides information regarding the location and characteristics of tissues, and these data are processed into grayscale images, on which the technology of ultrasound is based. ${ }^{10}$

Another important area of interest is the effect of various tissues/media upon the transmission of ultrasound waves. The term echogenicity refers to the ability of a tissue or medium to produce echoes by transmitting ultrasound waves in the context of surrounding tissues. ${ }^{11,12}$ When ultrasound waves pass through tissues with different echogenicity, the resultant images produced on the screen will differ in their contrast. Based on this, the images are categorized as hyperechoic (white on the screen), hypoechoic (gray on the screen), anechoic (black on the screen), and isoechoic (having echogenicity similar to that of a neighboring structure).

The generation and interpretation of ultrasound images vary from those of radiographic images because the energy sources differ widely. Modern ICU ultrasound machines are usually portable, lightweight, and have built-in memory to store images and videos.

To perform LUS, a high-frequency linear probe with a frequency of $5-12 \mathrm{MHz}$ is optimal because it provides better resolution and less penetration (Fig. 1A). For these reasons, this probe is ideal for imaging superficial structures like the pleura or for identifying lung sliding, and it is also effective in ultrasound-guided vascular procedures. A low-frequency curvilinear probe with a frequency of 3-5 MHz is useful in the assessment of effusions, consolidated lung, and the diaphragm because of good penetration and large sector width (Fig. 1B). A phased-array trans-

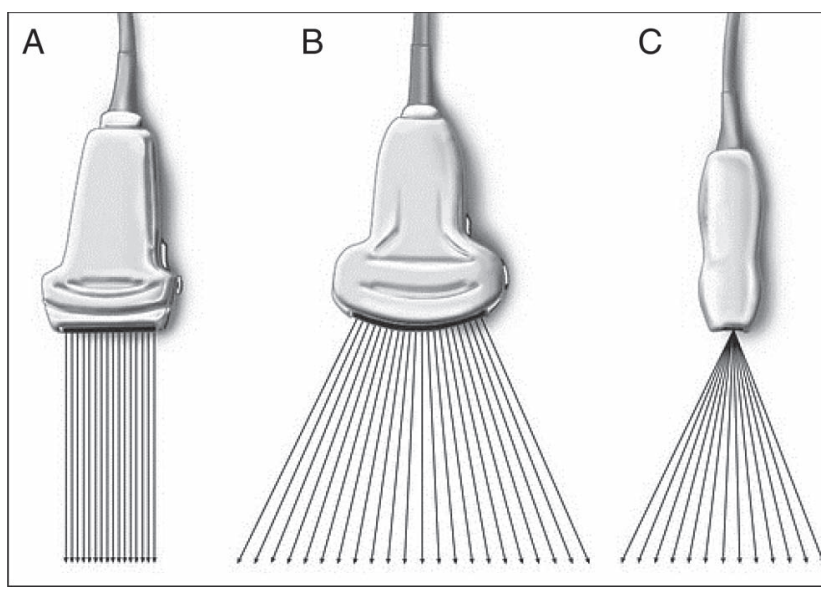

Fig. 1. Ultrasound probes commonly used in lung imaging. A: Linear array probe. B: Curved array probe. C: Phased array probe.

ducer probe with a frequency of $3-4.5 \mathrm{MHz}$ is also useful in LUS because the footprint can be placed easily in the intercostal spaces (Fig. 1C). These probes have a small footprint for fitting between the ribs to demonstrate all the signs of LUS, but the image clarity of the images is not as good compared to linear or curvilinear probes. ${ }^{13}$

Even though most ultrasound machines have uniform functional features, there remains some variation in the designs of different manufacturers. Hence it is important to become acquainted with the modes and controls of the specific machine before scanning. The control panel of an ultrasound machine has various controls that are used to adjust the quality of recording images. The commonly used modes while performing LUS are Bmode and M-mode.

B-mode, or brightness mode, is the basic mode of ultrasound imaging; it is also known as grayscale or 2-dimensional imaging, which refers to the standard blackand-white image obtained on the ultrasound monitor (Fig. 2A). M-mode, or motion mode, is used to visualize targets that are physically moving or to assess the movement of structures over time. The motion occurring in a 1-dimensional plane is displayed on the vertical axis, and time is displayed on the horizontal axis (Fig. 2B). The M-mode images are obtained by placing the cursor over the moving object on the B-mode image and activating the M-mode function. M-mode is very useful in the evaluation of cardiac valves and fetal heart activity. When speaking in terms of LUS, M-mode helps the clinician assess the respiratory variability of the inferior cava and, most importantly, identify pneumothorax. ${ }^{14}$ The lung and pleural structures are assessed using B-mode, whereas pleural movement is assessed with $\mathrm{M}$-mode. ${ }^{15}$

Currently there are no recommendations regarding the superiority of any specific mode for lung assessment. A specific mode or probe can be selected upon the lung 

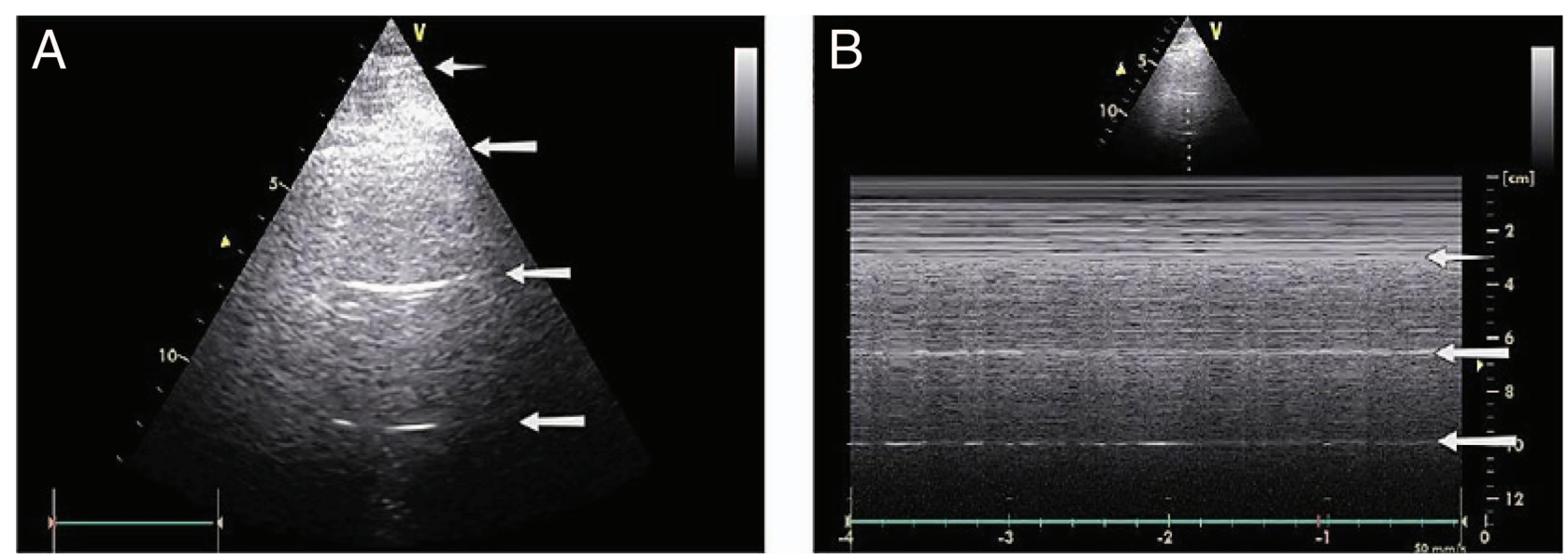

Fig. 2. Normal lung imaging, using B-mode and M-mode. A: B-mode illustrates A-lines. The A-lines or horizontal lines arising from the pleural line (arrow) are separated by regular intervals that are equal to the distance between the skin and the pleural line. B: M-mode shows the pleural line. Under the pleural line is the seashore sign (sandy pattern) due to the lung dynamics and pleural sliding. The horizontal lines are A-lines, separated by regular intervals (arrows). From Reference 110, with permission.

findings and clinician's discretion, with optimal settings recommended for lung imaging. ${ }^{16,17}$

\section{Clinical Applications of Lung Ultrasound}

As an imaging technique, LUS is easy to learn and simple to perform, and it has clear clinical utility in acute care medicine. Perhaps because bedside LUS in acute care is an essential technique for better patient outcomes, it required a pioneer from intensive care medicine, Dr Daniel Lichtenstein, to develop and implement this concept. LUS, a focused application of acute-care ultrasonography, helps clinicians rapidly diagnose and formulate immediate therapeutic plans related to lung disorders and pathologies. The LUS examination consists of 12 imaging zones, 6 on each side of thorax. The upper and basal part of each hemithorax is divided into anterior, lateral, and posterior zones, defined by the anterior and posterior axillary lines (Fig. 3). ${ }^{18}$ To master this imaging technique, one should have a clear understanding of the 7 principles and 10 basic signs of LUS, along with the required skills.

Following are the 7 principles of LUS, as described by Dr Lichtenstein in his work in $2004,{ }^{19}$ with an update on the seventh principle: 20

(1) A simple, unsophisticated ultrasound machine is adequate to perform LUS.

(2) Artifacts are generated in the thorax because gas and fluids have opposite locations or are mingled by pathologic processes.

(3) The lung is the most voluminous organ in the body with an extensive surface area (about $1,500 \mathrm{~cm}^{2}$ ). Thus, precise areas need to be defined.

(4) All lung signs arise from the pleural line.

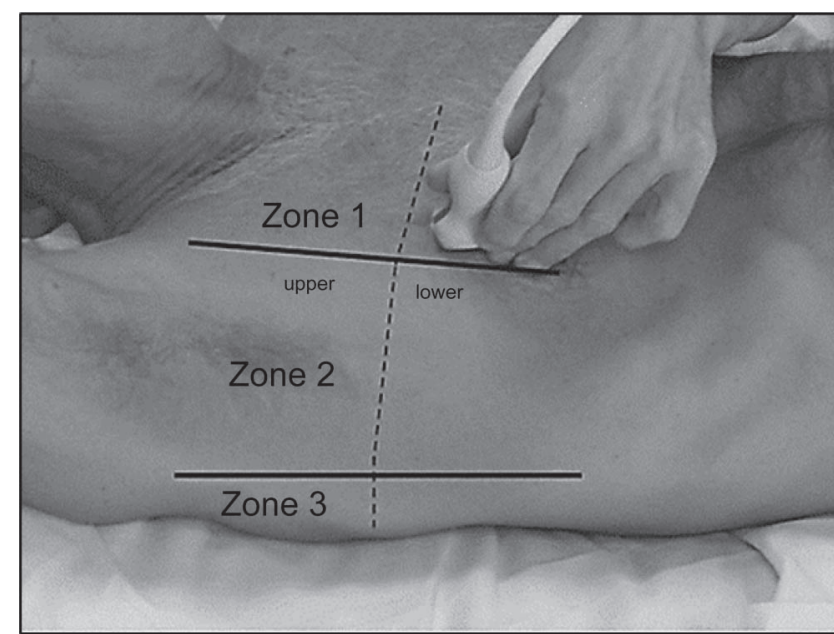

Fig. 3. Zones of lung ultrasound imaging. From Reference 18, with permission.

(5) LUS is largely based on the analysis of artifacts generated, and previously this was addressed as an issue to indicate the infeasibility of LUS.

(6) The lung is a vital organ and hence lung signs arising from the pleural line are dynamic

(7) A majority of acute, life-threatening lung disorders abut the pleural line, explaining the potential of LUS. Almost all life-threatening disorders, even those that may be less prominent in other tests, will show an extensive location with LUS, such as a pneumothorax, which can be small but still visible in a reasonably large projection..$^{20}$

LUS necessitates the understanding of the following 10 basic signs for interpreting underlying normal and ab- 


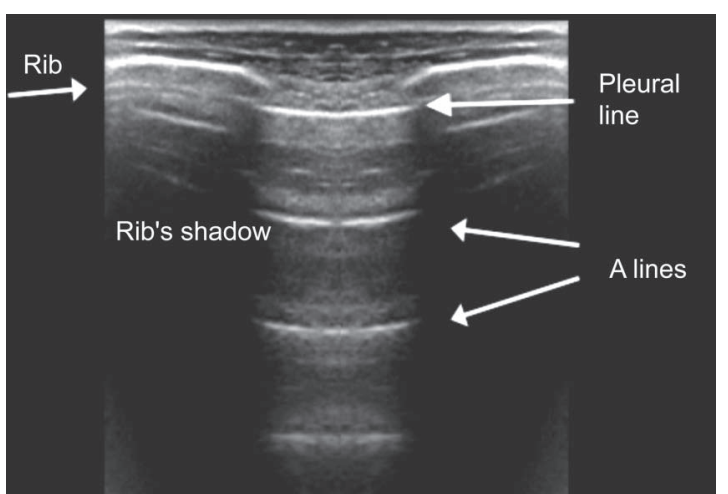

Fig. 4. Normal lung ultrasound image (B-mode) with the bat-wing sign and A-lines. From Reference 108, with permission.

normal lung conditions. ${ }^{13,21,22}$ These signs have been assessed in various studies and have diagnostic accuracies ranging from $90-100 \%$, which makes LUS a reasonable choice for the accepted bedside standard. ${ }^{23}$

For any ultrasound image obtained, a baseline image is required to correlate normal with abnormal. Similarly, the baseline normal image obtained during LUS is the pleural line/bat-wing sign. This baseline image is obtained in Bmode during initial lung screening. The pleural line lies $0.5 \mathrm{~cm}$ below the adjacent upper and lower rib shadows, yielding the appearance of a bat wing, with the ribs as the wings (Fig. 4). All LUS signs arise from the pleural line except for subcutaneous emphysema, where air under the skin will abolish the properties of ultrasound waves.

The second sign is the A-line or A-profile, which is a static sign seen in B-mode. Some of the ultrasound waves will bounce back and forth between the pleura and the transducer, generating reverberation artifacts called Alines. These fundamental, hyperechoic, reverberation artifacts of pleural lines are seen normally and are confirmatory for the presence of physiological or free air in thorax (Fig. 4).

Lung sliding is the third sign, and it is normal and dynamic in nature and is seen in B-mode at the same level of pleural line (Fig. 5, left). The parietal and visceral pleura are normally closely adhered with a minimal amount of fluid ( $<50 \mathrm{~mL}$ in healthy subjects) between them, allowing them to slide over one another during breathing. This appears as a backward and forward movement of the pleura. Lung sliding is synchronized with breathing, as the lungs descend toward the abdomen on inspiration. Lung sliding reflects the normal physiological movement of the lung toward the chest wall. This corresponds to the seashore sign in M-mode, which reflects the relation between lung sliding and superficial tissues (Fig. 5, right).

The fourth sign is the quad sign: it is an abnormal static LUS sign seen in B-mode, and it indicates the presence of a pleural effusion and is limited by 4 regular borders (Fig. $6 \mathrm{~A})$. The cephalic and caudal borders of a pleural effusion

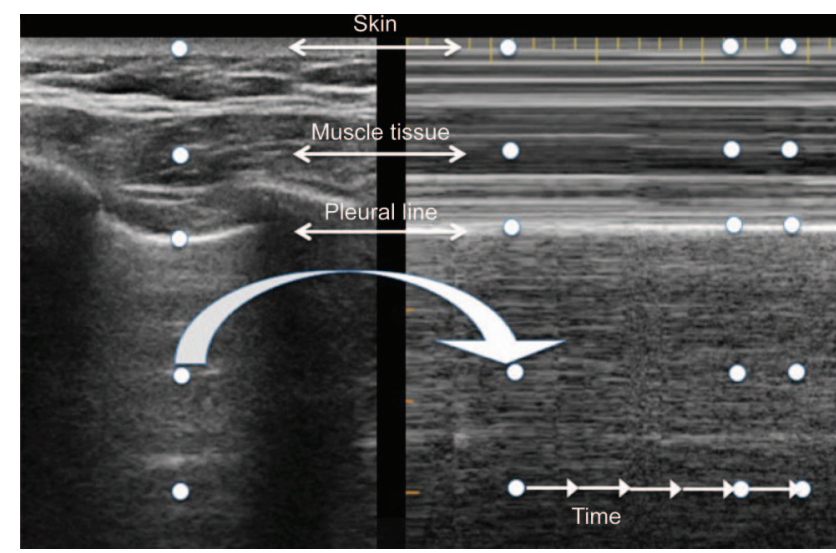

Fig. 5. Normal lung sliding image in B-mode (left) and the resultant normal seashore sign in M-mode (right). From Reference 108, with permission.
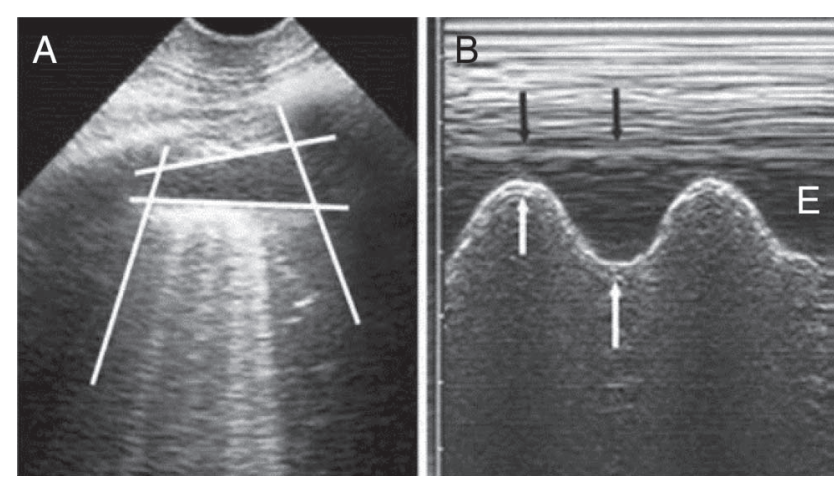

Fig. 6. Quad sign in B-mode and sinusoid sign in M-mode suggestive of pleural effusion. A: Below the pleural line, the regular line that is roughly parallel to the pleural line is the lung line, indicating the visceral pleura. This line, together with the pleural line and the shadow of the ribs, displays the quad sign. B: M-mode shows a movement of the lung line (white arrows) toward the pleural line (black arrows) on inspiration; the sinusoid sign, indicating a free pleural effusion and a viscosity enabling the use of a small-caliber needle if thoracentesis is envisaged. $\mathrm{E}=$ expiration. From Reference 21 , with permission.

are formed by the ribs at the top and the parietal and visceral pleura at the bottom. The deep boundary of the collection is regular, roughly parallel to the pleural line, and is called the lung line (ie, visceral pleura). If the image is obtained by excluding the diaphragm, the pleural effusion will appear as rectangular (ie, the quad sign), and in case of a significant effusion with the diaphragm visible, the basal lung can be seen floating in the irregular image of the effusion.

The fifth sign is the sinusoid sign, which is an abnormal dynamic LUS sign seen in M-mode, and it indicates the presence of a pleural effusion (Fig. 6B). This sign is displayed when the lung line moves toward the pleural line during inspiration. The sinusoid sign is obtained when the M-mode is activated by keeping the cursor in the center of 


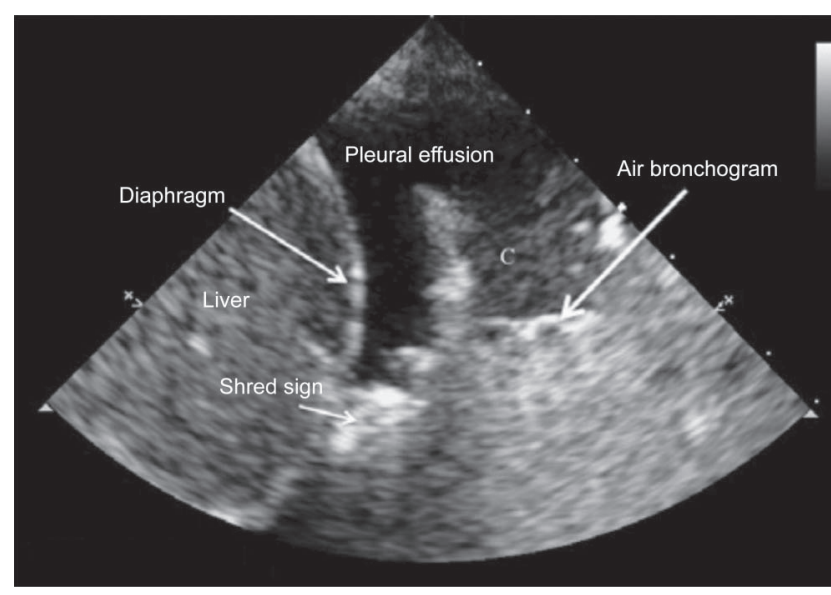

Fig. 7. Tissue-like sign and shred sign in B-mode, suggestive of consolidation. $\mathrm{C}=$ consolidated lung. From Reference 108, with permission.

the field with a large amount of pleural fluid as seen in B-mode. A sinusoid sign reinforces the clinician's findings that a pleural effusion is present and that the pleural fluid is not necessarily compromising lung dynamics.

The sixth sign mentioned is the shred sign, which is an abnormal sign seen in the B-mode. The shred sign represents non-translobar consolidation in most cases. The shred sign is formed when the border between consolidated and aerated lung is irregular, forming the fractal line, which is fully opposed to the lung line. This is a suggestive sign of pneumonia.

The seventh sign is the tissue-like sign, an abnormal sign that is observed in B-mode when there is a translobar consolidation (Fig. 7). When the lung is excessively filled with fluid, its echogenicity bears a resemblance to the liver, which is also called hepatization of lung.

The eighth sign, and one of the most important, is the B-line/lung rocket/comet tail artifacts. B-lines are seen in B-mode, and they are hyperechoic, long, vertical lines that originate from the pleural line and traverse the entire ultrasound screen, down to the bottom of the screen, erasing the normal A-lines (Fig. 8). These artifacts result from the mingling of air in the alveoli and water in the interlobular septa, due to the marked difference in acoustic impedance. The sound waves reverberate back and forth between the septa (because of the juxtaposition of air and water), creating a line for each reverberation that combine to form B-lines. The presence of B-lines is highly suggestive of alevolar-interstitial syndromes and pulmonary edema. Blines are also known as lung rockets or comet tail artifacts.

The ninth sign is absent lung sliding, which is an abnormal sign observed in the B-mode and is one of the 3 findings to confirm pneumothorax. The presence of air in the pleural space prevents the visualization of visceral pleura, and lung sliding will not be seen. To observe absent lung sliding, 2 adjacent ribs should be identified with

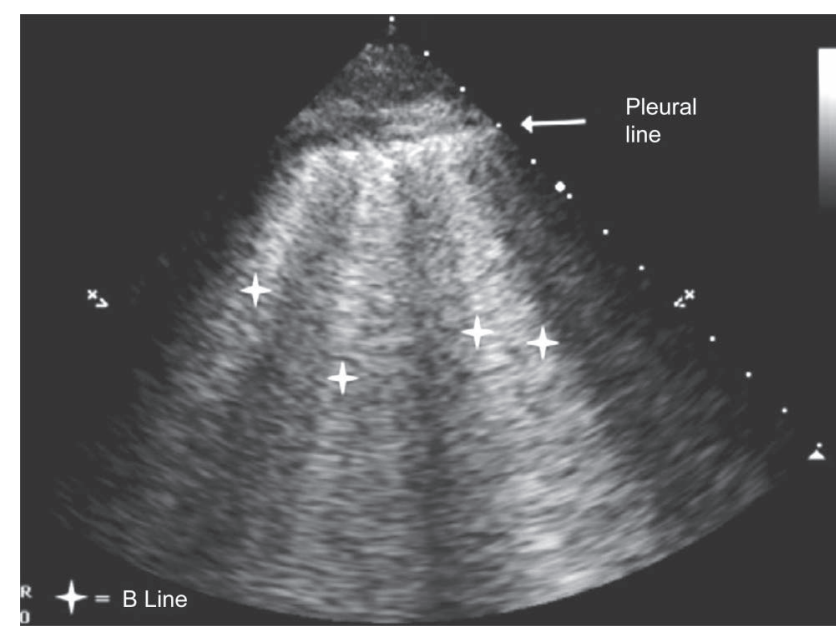

Fig. 8. B-lines/lung rockets/comet tail artifacts in B-mode, suggestive of alveolar interstitial syndrome/pulmonary edema. From Reference 108, with permission.

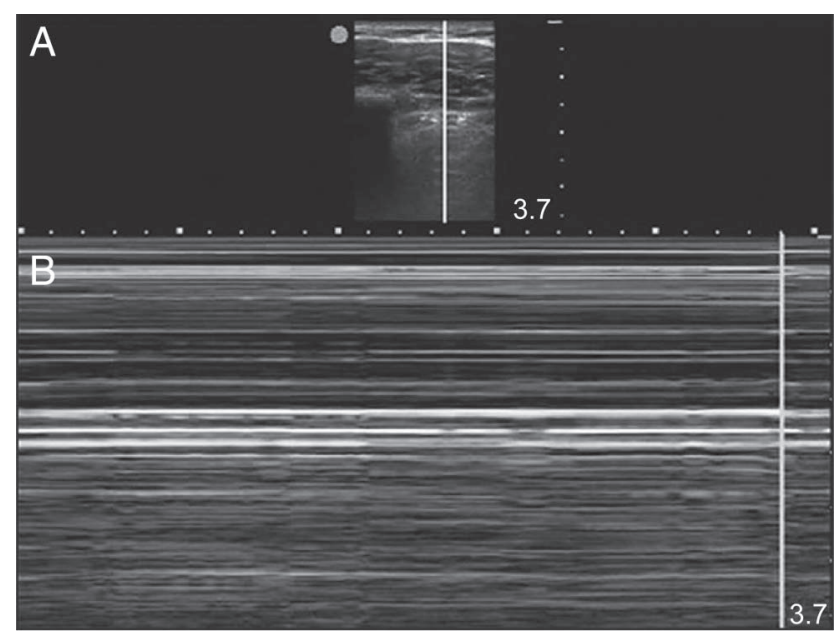

Fig. 9. Absent lung sliding image in B-mode $\mathrm{A}$ : and the resultant stratosphere sign/barcode sign in M-mode B:, suggestive of pneumothorax. From Reference 109, with permission.

the pleural line in between them. The typical back and forth movement of the pleural line with breathing will not be seen if there is any pleural involvement (pneumothorax, pleural effusion, pleurodesis) or lung involvement (pneumonectomy, pneumonia, lung intubation). ${ }^{13} \mathrm{M}$-mode is also used to confirm absent lung sliding. The resultant M-mode image in a pneumothorax will only display 1 pattern of parallel horizontal lines above and below the pleural line, demonstrating the lack of movement. This pattern bears a resemblance to a barcode and is often termed as barcode sign or stratosphere sign (Fig. 9).24,25

The tenth sign is the lung point, which is an abnormal sign seen in the M-mode. Lung point is the point at which the 2 pleural layers rejoin one another (Fig. 10). Lung point is considered as confirmatory for pneumothorax, pro- 


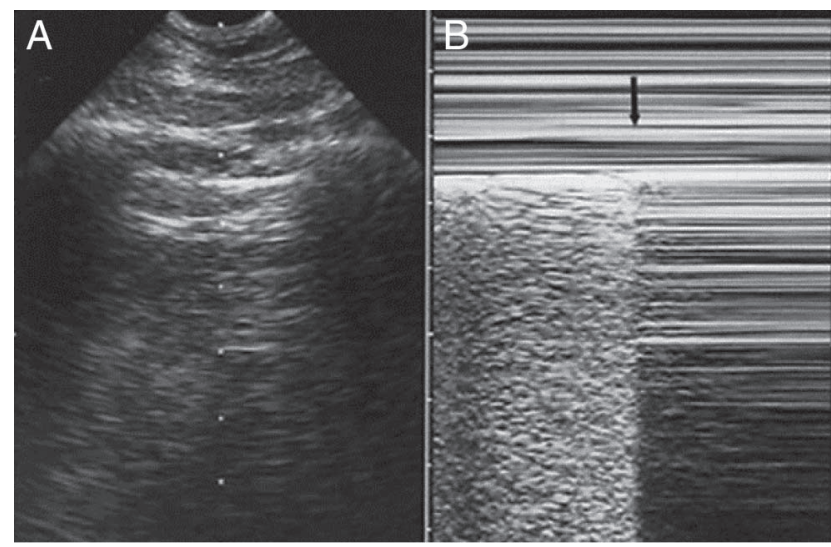

Fig. 10. Lung point, both in B-mode and M-mode, suggestive of pneumothorax. A: The pleural line just before the visceral pleura appears. B: The very moment the visceral pleura has touched the parietal pleural (arrow). This sign is called lung point; it can be seen along a line, but one point is sufficient for the diagnosis. From Reference 21, with permission.

vided lung sliding and B-lines are absent. ${ }^{13}$ This point will be visible by activating the M-mode and keeping the probe in the area at which the sliding is appearing and disappearing. Lung point represents the meeting point where lung inflation and deflation take place.

A literature review has revealed that, apart from these diagnostic approaches in ICUs, LUS is also helpful in managing ventilated patients, from confirmation of endotracheal intubation to initiation of mechanical ventilation and prediction of weaning outcome. ${ }^{26,27}$ The reduction of ventilator-related barotraumas through optimal PEEP and opening pressures are routinely practiced, ${ }^{28}$ whereby LUS can be a useful imaging tool for quantifying lung volume during mechanical ventilation, ${ }^{29}$ thereby preventing associated injuries.

It is necessary to mention some of the limitations of LUS, the most prominent being operator skill. This reinforces the fact that proper understanding and skills training in LUS is a prerequisite for reliable results. ${ }^{30}$ LUS also has patient-dependent limitations because it is difficult to examine obese patients using LUS due of the thickness of their rib cage, the presence of subcutaneous emphysema, or large thoracic dressings, any of which can alter or impede the transmission of sound waves to the lung periphery. It is also important to mention that LUS cannot detect hyperinflated lung fields that result from an increase in intrathoracic pressures. ${ }^{16}$ Another limitation is in the pediatric population, where LUS cannot address certain features, such as in a chest radiograph of a child presenting with respiratory distress, including hyperinflation and cardiac size and shape. ${ }^{31}$ To be specific and comprehensive, a LUS assessment should take approximately $15 \mathrm{~min}$, and with adequate knowledge and skills, clinicians can perform a lung examination more quickly. ${ }^{16}$

\section{Lung Ultrasound by Non-Physicians}

Although LUS requires only a short period of training, there remains considerable debate as to who should perform LUS. ${ }^{32}$ Long regarded as a technique to be performed by a physician, some recent studies ${ }^{33,34}$ suggest that the performance of LUS should be extended to other health care professionals, including physiotherapists and nurses. ${ }^{33,34}$ There is a paucity of literature that reflects the role and exposure of respiratory therapists (RTs) in LUS; we also looked into related areas such as respiratory physiotherapy and nursing, with an emphasis on their experience with LUS.

In the only available study that focused on LUS training for RTs, the authors concluded that RTs trained in ultrasound are independently capable of performing LUS with an accuracy of $>95 \% .35$ The authors designed a curriculum that covered both theory and practical applications of LUS. The theory portion was completed through lectures, internet resources, and self-study modules; the practical component was completed under direct supervision of an instructor. The authors demonstrated that upon completion of 10 supervised scans, $<2 \%$ of trainees required assistance with image acquisition, and $<5 \%$ of the images were interpreted incorrectly. Despite the fact that not all RTs had a chance to scan or diagnose pneumothorax because of fewer incidences, all of the RTs were able to independently identify normal images like A-lines or Aprofiles, thereby meeting the exclusion criteria of pneumothorax. In a short review of the abovementioned study, the reviewer mentioned that LUS training can be done in a short period of time with appropriate theoretical knowledge and practical demonstrations. The review also identified further need of clinical outcomes data on LUS performed by RTs. ${ }^{36}$

In one case study, the authors mentioned that the addition of LUS helped determine the diagnosis of pleural effusion in a symptomatic and deteriorating patient, after a chest radiograph and auscultation did not. Chest physiotherapy and bronchial hygiene therapies were tried initially, yet LUS aided in the diagnosis of pleural effusion and drainage of pleural fluid, thereby preventing intubation. ${ }^{37}$ In a conference proceeding, 4-phase LUS training was used to train RTs to use the technique independently, ${ }^{38}$ and still another review concluded that the superior sensitivity and specificity of LUS would help critical care clinicians reach an accurate, point-of-care diagnosis of respiratory disorders, enabling them to plan the need and determinants of respiratory care maneuvers. ${ }^{33}$ Another narrative review also found that LUS could be an additional advantage alongside other assessment modalities to guide, monitor, and evaluate the effectiveness of chest physiotherapy. ${ }^{39}$ 
Literature reviews have demonstrated that nurse-performed LUS is also accurate in identifying cardiorespiratory derangements. In one study, the authors assessed nurseperformed LUS in diagnosing cardiogenic dyspnea, and they found a sensitivity and specificity of $>95 \% .{ }^{40}$ The authors concluded that nurses with LUS training can accurately categorize patients with cardiogenic or non-cardiogenic dyspnea in emergency departments. Another study suggested that nurse-performed LUS may be a potentially useful alternative to the traditional physician-performed LUS. They observed good accuracy in diagnosing cardiogenic dyspnea and summarized that nurse-performed LUS, in combination with brain natriuretic peptide, is useful in ruling out dyspnea of cardiogenic origin..$^{41}$

In contrast, another study that looked into the feasibility of paramedic-performed prehospital LUS found this approach to be nonsignificant because $>50 \%$ of the scanned images were deemed uninterpretable upon expert review. ${ }^{42}$

\section{The Emerging Role of Respiratory Therapists}

RTs possess the knowledge, skill, and ability to provide a wide range of diagnostic and therapeutic procedures to patients who require basic, advanced, and prolonged cardiopulmonary and related services. In addition, RTs are most advantageous in improving patient outcomes and thereby reducing morbidities. ${ }^{43,44}$ An example of their advancement in professional practice is the presence of qualified RTs in therapeutically hybrid areas like extracorporeal life-support therapy. ${ }^{45-47}$ It can be seen that ICUs around the world are implementing standard guidelines and protocols for mechanical ventilation, and this empowers RTs to be involved in ventilator management, including the selection of ventilator mode, determining the ventilation strategy, and participating in the decision to wean. ${ }^{48,49}$ Many randomized, controlled studies have concluded that RT-driven mechanical ventilation protocols result in shorter weaning times, increased successful extubation rate, and ventilator-free days, as well as a reduction in overall morbidities. ${ }^{50,51}$ An extended clinical role for RTs was demonstrated when a prospective LUS training study and subsequent review highlighted that RTs who are naïve in ultrasonography could be trained to perform LUS independently and competently, with a post-training accuracy of $>95 \% .34,35$

Because RTs are mainly involved in the care of acutely ill patients, we reviewed the scope of lung, diaphragmatic, and airway ultrasound in the following applied areas, where their expertise can be widely utilized.

\section{Recognition of Pneumothorax}

Of all the ventilator-associated complications, pneumothorax was found to be an independent predictor of mor- tality during mechanical ventilation..$^{52}$ Pneumothorax was also associated with a significant increase in morbidities such as increased length of ICU and hospital stay..$^{53}$ Pneumothorax is rarely seen in ventilated patients with normal lungs, and it is most often seen in patients with underlying respiratory pathology, such as obstructive airway diseases and ARDS. ${ }^{54-56}$ The main causative factors are inappropriate PEEP and barotrauma. ${ }^{54,57,58}$ Many of the clinical subjective and objective findings are nonspecific and are not considered to be reliable indicators of pneumothorax..$^{59}$ Imaging techniques remain the accepted standard for the recognition of pneumothorax. ${ }^{60}$

In the ICU, most patients are in a supine or semi-recumbent position, and anterior-posterior chest radiograph films are often obtained, limiting the reliability of chest radiographs. ${ }^{61,62}$ Although computed tomography is considered to be the optimal choice, it is not always practical or safe to move a hemodynamically unstable, ventilated patient to the radiology department to exclude pneumothorax. ${ }^{6}$ Hence, the practice of LUS for the diagnosis of pneumothorax has emerged as the method of choice with clinicians who can perform bedside ultrasonography. ${ }^{63,64}$ Acute worsening of ventilated patients with cardiorespiratory compromise is a challenging scenario in ICU practice. The mnemonic DOPE is often used to identify the cause, where $\mathrm{D}$ stands for dislodgement of the endotracheal tube, $\mathrm{O}$ for obstruction of tube, $\mathrm{P}$ for pneumothorax, and $\mathrm{E}$ for equipment failure. ${ }^{65}$ Of these 4 concerns, all except pneumothorax are recognizable and manageable at the bedside without much delay. It is understood that management of pneumothorax is often delayed due to the lack of prompt recognition. The ultrasound features of pneumothorax includes abolished lung sliding, absence of B-lines, absence of the lung pulse, and presence of the lung point. ${ }^{66}$ The sequential assessment of pneumothorax starts with looking for the presence of lung sliding on the highest point of the anterior chest, which can exclude pneumothorax within seconds. If lung sliding is absent, then the presence of $\mathrm{B}$-lines is checked to rule out pneumothorax. If there is no sliding and no B-lines, then the lung point should be sought, which confirms the presence of pneumothorax. Basic training with a 5-min online video showed a high accuracy in giving clinicians knowledge to exclude pneumothorax, and that this knowledge was retained for at least 6 months. ${ }^{67}$

RTs, being one of the primary practitioners of mechanical ventilation, play an important role in the identification and rectification of ventilator-related problems, and LUS may specifically facilitate their diagnostic skills in identifying pneumothorax.

\section{Weaning from Mechanical Ventilation}

Alternatively of interest for RTs is the utility of ultrasound in predicting weaning outcome. Weaning or 
liberation from mechanical ventilation is a complex, multifactorial process with a failure rate of approximately 20\%. ${ }^{68}$ Diaphragmatic ultrasound provides a direct and rapid assessment of diaphragmatic movement and function because it is a prime indicator of a patient's spontaneous efforts.

It is of clinical interest whether diaphragmatic ultrasound can predict successful weaning. In a prospective cohort, the authors used diaphragmatic ultrasound to assess mean diaphragmatic thickness (mean of daily assessed end-expiratory diaphragmatic thickness), diaphragmatic thickness fraction (thickness at end inspiration - thickness at end expiration/thickness at end expiration $\times 100$ ), mean diaphragmatic excursion (average of diaphragmatic excursion during tidal breathing), and diaphragmatic dystrophy (diaphragmatic thickness $<2 \mathrm{~mm}$ ). They observed that all of these variables were significantly decreased with the increased length and duration of mechanical ventilation, thereby concluding that diaphragmatic ultrasound is a sensitive tool for predicting weaning outcome. ${ }^{69}$

In another study, investigators examined ultrasound assessment of diaphragmatic movement and lung aeration, and they concluded that these techniques were superior for predicting the weaning process in comparison to the traditional indices related to blood gases and respiratory mechanics. ${ }^{70}$ The loss of lung volume after extubation is a hallmark sign of extubation failure, leading to poor gas exchange, prolonged mechanical ventilation, and increased mortality and morbidity. ${ }^{71}$ Lung aeration loss can be estimated using a validated score called the Lung Ultrasound Score. The LUS score of aeration is calculated for each given region of interest. Points are allocated according to the worst ultrasound pattern observed: normal aeration $=0$, well separated B-lines $=1$, coalescent B-lines $=2$, and consolidation $=3$ (Fig. 11). ${ }^{26}$ A LUS score ranging between 0 and 36 was calculated as the sum of each region. ${ }^{72}$ The authors concluded that the LUS score of aeration changes during a successful spontaneous breathing trial can accurately predict postextubation distress. They also concluded that a LUS score of $<13$ predicts successful extubation, whereas a LUS score of $>17$ is considered a threshold for postextubation distress and extubation failure. Because RT-driven weaning protocols are accepted globally, the addition of diaphragmatic ultrasound screening is likely to help in predicting successful weaning and extubation.

\section{Airway Management}

RTs participate as key members of airway management teams, where their expertise is appreciated from basic bagvalve-mask ventilation to advanced techniques related to difficult intubations. Airway imaging is a newer application of ultrasonography that is proven to lead to better
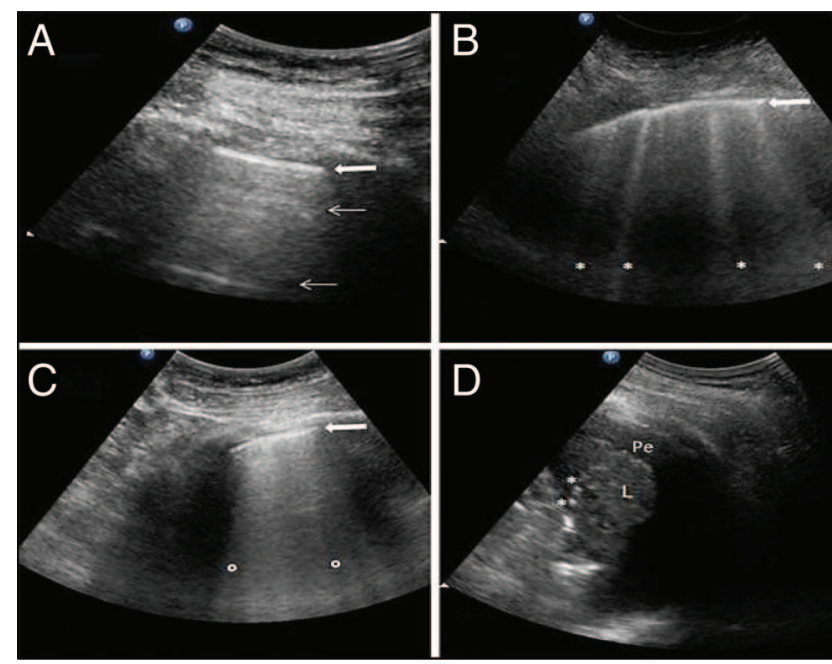

Fig. 11. Lung ultrasound patterns corresponding to progressive loss of aeration. A: Normal pattern with multiple horizontal A-lines. $B$ : The pleural line is visible with separated B-lines, suggestive of moderate lung aeration loss. C: The pleural line is visible with coalescent B-lines arising from the pleural line, suggestive of severe in lung aeration loss. D: Transverse view of a consolidated lower lobe. From Reference 26, with permission.

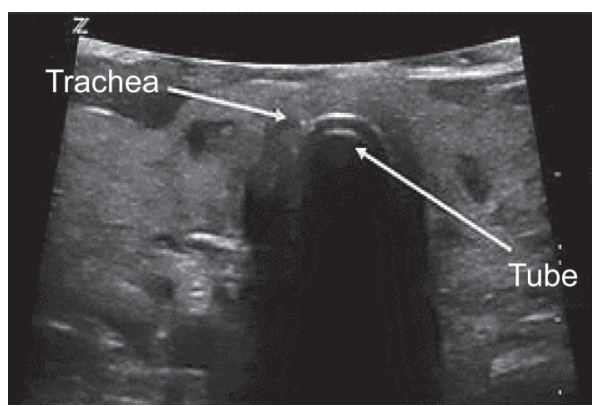

Fig. 12. Ultrasound image of endotracheal intubation, with semicircular hyperechogenicity. From Reference 83, with permission.

patient outcome. ${ }^{73-75}$ Airway ultrasound is useful in various areas of RTs' involvement in airway management, including confirmation of endotracheal tube placement, ${ }^{75,76}$ prediction of difficult intubation, ${ }^{77}$ prediction of pediatric endotracheal tube size and placement, ${ }^{78,79}$ detection of subglottic stenosis,, 80 prediction of postextubation stridor, ${ }^{81}$ and confirmation of laryngeal mask airway position. ${ }^{82} \mathrm{Ul}-$ trasound confirmation of endotracheal intubation is achieved by keeping the transducer at the level of the suprasternal notch in the transverse approach, where the shadow of the endotracheal tube is seen as semicircular hyperechoic lines in the trachea with an empty esophagus (Fig. 12). ${ }^{83}$ Positioning the linear probe transversely over the cricothyroid membrane during the intubation process results in confirmatory reverberation artifacts, known as the bullet sign. ${ }^{84}$ Similarly, esophageal intubation is detected by a double tract sign (Fig. 13), whereby the esoph- 


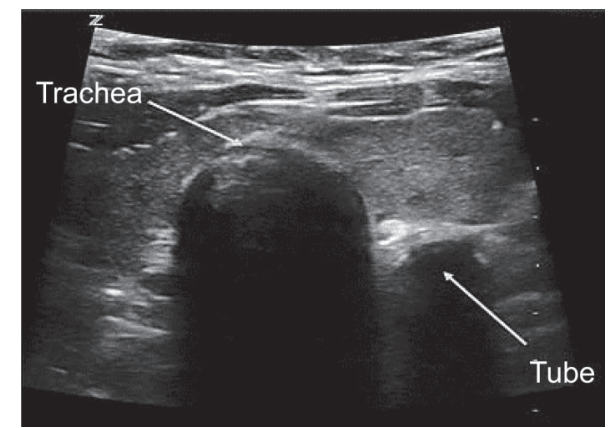

Fig. 13. Ultrasound image of esophageal intubation, with double tract sign. From Reference 83, with permission.

agus will be distended with the endotracheal tube inside. Both the tracheal and the esophageal tracts are seen in Figure 13, indicating esophageal intubation. ${ }^{83}$

A systematic review and meta-analysis concluded that trans-tracheal ultrasound confirms endotracheal intubation with superior sensitivity and specificity. The review also confirmed this technique's utility in emergency intubations as an initial assessment before the final confirmation with capnography. ${ }^{85}$ Yet another recent prospective cohort concluded that airway ultrasound, end-tidal capnography, and a conventional clinical method of 5-point auscultation have comparable sensitivity and specificity in identifying tracheal or esophageal position of the endotracheal tube. The authors also confirmed the clinical importance of ultrasonographic assessment because the time difference in confirming endotracheal tube placement was observed to be significantly faster than the other 2 methods. ${ }^{86}$ As advocates for patient safety during airway management, RTs could justify extending their scope of practice with airway ultrasound to enhance patient safety and reduce adverse events.

\section{Competency and Training in Lung Ultrasound}

A review of the literature shows that, although some researchers have attempted to standardize the essential skills to attain competency in LUS among ICU physicians, the duration of training needed remains unclear. ${ }^{87,88}$

It is also evident that the ultrasound training of mid-level providers and other non-physicians in developing countries is relatively inadequate. The utility, however, is growing, ${ }^{89-91}$ and it is suggested that, if educated, non-physician professionals would be able to adopt ultrasonography into their clinical practice and would utilize it frequently. ${ }^{92}$

A significant difference is observed in the duration and methodology of LUS training programs that are currently available. It has been observed from a number of small studies and conference abstracts that LUS skills can be satisfactorily attained with anywhere from $2 \mathrm{~h}$ to 4 months of training, and with $20-80$ supervised scans. ${ }^{93-98}$
Some authors have suggested the need for a minimum of 100 chest ultrasound procedures ${ }^{99}$ to confirm competency, whereas others have emphasized a duration of LUS training for acceptable competency ranging from $30 \mathrm{~min}^{94}$ to 7 months. ${ }^{100}$ One study proposed performing several LUS examinations on a daily basis to help clinicians become competent in identifying pleural effusion, lung consolidation, and alveolar interstitial syndrome within 6 weeks. ${ }^{16}$ However, the authors did not specify the number of scans required.

In a recent publication, a new scale, the Assessment of Competency in Thoracic Sonography, was introduced to assess the quality of point-of-care thoracic ultrasound performed by new learners. The authors concluded that the scale was effective in making valid judgments regarding the competency of novices in point-of-care thoracic ultrasound, and the majority of learning improvement occurred in their first $25-30$ practice studies. ${ }^{101}$

Another publication on competency assessment of LUS was the LUS-Objective Structured Assessment of technical skills. The 26-element competency check includes 6 domains: indication for LUS examination, systematic approach, technical skills, recognition and differentiation of normal anatomy from pathology, documentation and reporting the examination, and diagnostic conclusion. The authors concluded that this assessment tool provides a relevant, valid, and feasible method to assess the competency of operators from diverse medical specialties. ${ }^{102}$

While there is a learning curve associated with the application of LUS, it is relatively short, ${ }^{103}$ and the diagnostic yield of LUS largely depends on the performer's expertise. ${ }^{104-106}$ A consensus process was aimed at unifying the approach and language of 6 major areas of LUS (ie, terminology, technology, technique, clinical outcomes, cost effectiveness, and future research) to obtain LUS effectiveness in various clinical settings, such as acute care areas, operating rooms, and out-patient clinics. They advocated LUS as a point-of-care tool, with training attained through the combination of theory and bedside mentoring. ${ }^{107}$

Because LUS is not routinely used by RTs in clinical practice, the level of training, the number of scans, and the duration of training required to achieve competency in LUS is unknown. Considering the vast scope of practice and intended positive outcomes, RTs can be utilized for performing LUS subsequent to adequate training and competency assessment. Hence, it is highly suggested that professional respiratory care bodies of various countries initiate training programs for LUS among RTs with competency certification.

\section{Conclusion}

It is apparent from this review that the potential role of RTs in imaging areas, specifically LUS, is vast but underutilized. The in-depth knowledge and skills possessed by 
RTs, acquired throughout their academic and clinical careers, provide an ideal foundation for them to identify and manage any cardiorespiratory emergency. We are well acquainted with the range of RTs' responsibilities, and we therefore believe RTs should be trained in LUS. Acquiring this skill is a benchmark in current practice, and it may assist RTs in reducing adverse events in ventilated patients. Lung, diaphragmatic, and airway ultrasound appear to be closely related and promising tools for RTs' because they offer better diagnostic accuracy in resource- and timelimited settings. It also appears to be a clinical technology that is easily taught.

Foreseeing professional advancement and better patient outcomes, we recommend the inclusion of a comprehensive respiratory care-related ultrasound training module within the existing worldwide respiratory therapy curriculum. We also suggest a well-structured respiratory care protocol related to ultrasound training for working RTs, to augment their technical and clinical decision-making skills for safer practice.

\section{Directions of Future Research}

This review was aimed at exploring the potential for respiratory therapists to perform LUS, as well as diaphragmatic and airway ultrasound. However, there are substantial gaps identified from the current available literature regarding RTs' exposure to diagnostic ultrasound, although it clearly has an influence on respiratory care practices. Future research focusing on randomized, controlled, academic and clinical trials using diagnostic ultrasound as a part of a respiratory therapist's clinical practice is highly recommended. The need for prospective studies to explore training requirements for respiratory therapists to attain competency in lung, diaphragmatic and airway ultrasound is also suggested. Such studies may subsequently reflect the scope of inclusion of diagnostic ultrasound in respiratory care services. Conducting randomized clinical trials on patient outcomes is challenging, hence alternative methods of demonstrating efficacy, such as comparative accuracy and decision making studies, may also be explored.

We therefore emphasize that the wide clinical scope of diagnostic ultrasound, and the lack of its related literature in the respiratory care profession, as reflected in our review should be considered an eye opener for respiratory therapists to get involved in the training and subsequent practice of this imaging tool.

\section{ACKNOWLEDGMENT}

The authors thank all of the staff members of the Department of Respiratory Services, Sidra Medicine, especially Jennifer Piette and Shafina Eddicott, for their constant support in preparing this review. The authors also thank all of the teachers in the field of respiratory therapy, without whom respiratory therapists would not have succeeded in their professional achievements.

\section{REFERENCES}

1. Henscke CI, Pasternack GS, Schoroeder S, Hart KK, Herman PG. Bedside chest radiography. Diagnostic efficacy. Radiology 1983; 149(1):23-26.

2. Greenbaum DM, Marschall KE. The value of routine daily chest $\mathrm{X}$-rays in intubated patients in the medical intensive care unit. Crit Care Med 1982;10(1):29-30.

3. Bekemeyer WB, Crapo RO, Calhoon S, Cannon CY, Clayton PD. Efficacy of chest radiography in a respiratory intensive care unit. A prospective study. Chest 1985;88(5):691-696.

4. Rouby JJ, Puybasset L, Cluzel P, Richecoeur J, Lu Q, Grenier P. Regional distribution of gas and tissue in acute respiratory distress syndrome. II. Physiological correlations and definition of an ARDS Severity Score. CT Scan ARDS Study Group. Intensive Care Med 2000;26(8):1046-1056.

5. Oba Y, Zaza T. Abandoning daily routine chest radiography in the intensive care unit. Meta-analysis. Radiology 2010;255(2):386-395.

6. Beckmann U, Gillies DM, Berenholtz SM, Wu AW, Pronovost P. Incidents relating to the intra-hospital transfer of critically ill patients. An analysis of the reports submitted to the Australian Incident Monitoring Study in Intensive Care. Intensive Care Med 2004; 30(8):1579-1585.

7. Xirouchaki N, Kondili E, Prinianakis G, Malliotakis P, Georgopoulos D. Impact of lung ultrasound on clinical decision making in critically ill patients. Intensive Care Med 2014;40(1):57-65.

8. Hangiandreou NJ. AAPM/RSNA physics tutorial for residents. Topics in US: B-mode US: basic concepts and new technology. Radiographics 2003;23(4):1019-1033.

9. Aldrich JE. Basic physics of ultrasound imaging. Crit Care Med 2007;35(5 Suppl):S131-S137.

10. Anantham D, Ernst A. Ultrasonography. In: Mason RJ, Broaddus VC, Murray JF, Nadel JA, editors. Murray and Nadel's textbook of respiratory medicine. Philadelphia: Saunders-Elsevier; 2010:445460.

11. Bigeleisen PE, Orebaugh SL. Principles of Sonography. In: Bigeleisen PE, editor. Ultrasound-guided regional anesthesia and pain medicine. Philadelphia: Lippincott Williams and Wilkins; 2010:10-25.

12. Bakhru RN, Schweickert WD. Intensive care ultrasound: I. Physics, equipment, and image quality. Ann Am Thorac Soc 2013;10(5): 540-548.

13. A Miller. Practical approach to lung ultrasound. BJA Educ 2016; 16(2):39-45.

14. Markowitz J. Probe selection, machine controls, and equipment. In: Carmody KA, Moore CL, Feller-Kopman D, editors. Handbook of critical care and emergency ultrasound. New York: McGraw-Hill; 2011:25-38.

15. Chichra A, Makaryus M, Chaudhri P, Narasimhan M. Ultrasound for the pulmonary consultant. Clin Med Insights Circ Respir Pulm Med 2016;10:1-9.

16. Bouhemad B, Zhang M, Lu Q, Rouby JJ. Clinical review: Bedside lung ultrasound in critical care practice. Crit Care 2007;11(1):205.

17. Via G, Storti E, Gulati G, Neri L, Mojoli F, Braschi A. Lung ultrasound in the ICU: From diagnostic instrument to respiratory monitoring tool. Minerva Anestesiol 2012;78(11):1282-1296.

18. Doerschug KC, Schmidt GA. Intensive care ultrasound: III. Lung and pleural ultrasound for the intensivist. Ann Am Thorac Soc 2013;10(6):708-712.

19. Lichtenstein D. Lung ultrasound in the critically ill. In JL Vincent, editor. Yearbook of intensive care and emergency medicine. Heidelberg: Springer; 2004:625-644. 


\section{Lung UltRASOUND By RESPIRATORY THERAPISTS}

20. Lichtenstein D. Novel approaches to ultrasonography of the lung and pleural space: where are we now? Breathe 2017;13(2):100-111.

21. Lichtenstein DA. Lung ultrasound in the critically ill. Ann Intensive Care 2014;4(1):1.

22. Lichtenstein DA. An Introduction to the signatures of lung ultrasound. In: Lichtenstein DA, editor. Lung ultrasound in the critically ill. Switzerland: Springer, Cham; 2016:59-60.

23. Lichtenstein DA. BLUE-protocol and FALLS-protocol: two applications of lung ultrasound in the critically ill. Chest 2015;147(6): 1659-1670.

24. Barillari A, Kiuru S. Detection of spontaneous pneumothorax with chest ultrasound in the emergency department. Intern Emerg Med 2010;5(3):253-235.

25. Johnson A. Emergency department diagnosis of pneumothorax using goal-directed ultrasound. Acad Emerg Med 2009;16(12):13791380.

26. Bouhemad B, Mongodi S, Via G, Rouquette I. Ultrasound for "lung monitoring" of ventilated patients. Anesthesiology 2015;122(2): 437-447.

27. Pecanha Antonio A, Souza Castro P, Schulz L, Maccari J, Oliveira R, Teixeira C, Knorst M. Lung ultrasound findings predict weaning failure from mechanical ventilation. Crit Care 2014;18(1):298.

28. Gattinoni L, Caironi P, Cressoni M, Chiumello D, Ranieri VM, Quintel M, et al. Lung recruitment in patients with the acute respiratory distress syndrome. N Engl J Med 2006;354(17):1775-1786.

29. Corradi F, Brusasco C, Vezzani A, Santori G, Manca T, Ball L, et al. Computer-aided quantitative ultrasonography for detection of pulmonary edema in mechanically ventilated cardiac surgery patients. Chest 2016;150(3):640-651.

30. Kreuter M, Mathis G. Emergency ultrasound of the chest. Respiration 2014;87(2):89-97.

31. Tomà $\mathrm{P}$, Owens CM. Chest ultrasound in children: critical appraisal. Pediatr Radiol 2013;43(11):1427-1434.

32. Cantinotti M, Giordano R, Valverde I. Lung ultrasound: a new basic, easy, multifunction imaging diagnostic tool in children undergoing pediatric cardiac surgery. J Thoracic Dis 2017;9(6):13961399.

33. Leech M, Bissett B, Kot M, Ntoumenopoulos G. Lung ultrasound for critical care physiotherapists: a narrative review. Physiother Res Int 2015;20(2):69-76.

34. Vitale J, Mumoli N, Giorgi-Pierfranceschi M, Cresci A, Cei M, Basile $\mathrm{V}$ et al. Comparison of the accuracy of nurse-performed and physician-performed lung ultrasound in the diagnosis of cardiogenic dyspnea. Chest 2016;150(2):470-471.

35. See KC, Ong V, Wong SH, Leanda R, Santos J, Taculod J, Phua J, Teoh CM. Lung ultrasound training: curriculum implementation and learning trajectory among respiratory therapists. Intensive Care Med 2016;42(1):63-71.

36. Wong A. How much specialised lung ultrasound training is needed for RTs? European Society of Intensive Care Medicine News: Jan 11, 2016. Available at: https://www.esicm.org/icm-article-reviewspecialised-lung-ultrasound-training-wong/. Accessed Dec 122017.

37. Leech M, Bissett B, Kot M, Ntoumenopoulos G. Physiotherapistinitiated lung ultrasound to improve intensive care management of a deteriorating patient and prevent intubation: a case report. Physiother Theory Pract 2015;31(5):372-376.

38. Hayward S, Kelly D. Respiratory physiotherapy and lung ultrasound: a service evaluation of a training programme. ICS State of the Art; e-posters: Dec 2016. Available at: http://epostersonline.com/ soa2016/node/167. Accessed Dec 152017.

39. Le Neindre A, Mongodi S, Philippart F, Bouhemad B. Thoracic ultrasound: potential new tool for physiotherapists in respiratory management. A narrative review. J Crit Care 2016;31(1):101-109.
40. Unluer EE, Karagoz A, Oyar O, Vandenberk N, Kiyançiçek S, Budak F. Lung ultrasound by emergency nursing as an aid for rapid triage of dyspneic patients: a pilot study. Int Emerg Nurs 2014; 22(4):226-231.

41. Mumoli N, Vitale J, Giorgi-Pierfranceschi M, Cresci, A. Cei M, Basile V, et al. Accuracy of nurse-performed lung ultrasound in patients with acute dyspnea: a prospective observational study. Medicine 2016;95(9):e2925.

42. Becker TK, Martin-Gill C, Callaway CW, Guyette FX, Schott C. Feasibility of paramedic performed prehospital lung ultrasound in medical patients with respiratory distress. Prehosp Emerg Care 2018; 22(2):175-179.

43. Stoller JK. 2000 Donald F. Egan Scientific Lecture. Are respiratory therapists effective? Assessing the evidence. Respir Care 2001; 46(1):56-66

44. Stoller JK. The effectiveness of respiratory care protocols. Respir Care 2004;49(7):761-765.

45. Moll V, Teo EYL, Grenda DS, Powell DC, Connor MJ, Gartland BT. et al. Rapid development and implementation of an ECMO program. ASAIO J 2016;62(3):354-358.

46. The ECMO Team as recommended by Extracorporal Life Support Organization, ELSO Resources 2017. Available at: https://www. elso.org/Resources/TheECMOTeam.aspx. Accessed Dec 132017.

47. Position statement as recommended by American Association of Respir Care, AARC Resources 2017. Available at: https:// www.aarc.org/wp-content/uploads/2017/03/statement-of-ecmo.pdf. Accessed Dec 132017.

48. Haas CF, Loik PS. Ventilator discontinuation protocols. Respir Care 2012;57(10):1649-1662.

49. Ellis SM, Dainty KN, Munro G, Scales DC. The use of mechanical ventilation protocols in the intensive care unit: a survey of current practice. Crit Care 2012;27(6):556-563.

50. Gupta P, Giehler K, Walters RW, Meyerink K, Modrykamien AM. The effect of a mechanical ventilation discontinuation protocol in patients with simple and difficult weaning: impact on clinical outcomes. Respir Care 2014;59(2):170-177.

51. Scheinhorn DJ, Chao DC, Stearn-Hassenpflug M, Wallace WA. Outcomes in post-ICU mechanical ventilation: a therapist-implemented weaning protocol. Chest 2001;119(1):236-242.

52. Esteban A, Anzueto A, Frutos F, Alía I, Brochard L, Stewart TE, et al. Characteristics and outcomes in adult patients receiving mechanical ventilation: a 28-day international study. JAMA 2002; 287(3):345-355.

53. de Lassence A, Timsit JF, Tafflet M, Azoulay E, Jamali S, Vincent F, et al. Pneumothorax in the intensive care unit: incidence, risk factors, and outcome. Anesthesiology 2006;104(1):5-13.

54. Petersen GW, Baier H. Incidence of pulmonary barotrauma in a medical ICU. Crit Care Med 1983;11(2):67-69.

55. Pierson DJ. Complications associated with mechanical ventilation. Crit Care Clin 1990;6(3):711-724.

56. Parker JC, Hernandez LA, Peevy KJ. Mechanisms of ventilatorinduced lung injury. Crit Care Med 1993;21(1):131-143.

57. Schnapp LM, Chin DP, Szaflarski N, Matthay MA. Frequency and importance of barotraumas in 100 patients with acute lung injury. Crit Care Med 1995;23(2):272-278.

58. Gattinoni L, Bombino M, Pelosi P, Lissoni A, Pesenti A, Fumagalli $\mathrm{R}$, Tagliabue M. Lung structure and function in different stages of severe adult respiratory distress syndrome. JAMA 1994;271(22): 1772-1779.

59. Hsu C-W, Sun S-F. Iatrogenic pneumothorax related to mechanical ventilation. World J Crit Care Med 2014;3(1):8-14.

60. Rankine JJ, Thomas AN, Fluechter D. Diagnosis of pneumothorax in critically ill adults. Postgrad Med J 2000;76(897):399-404. 


\section{Lung UltRASOUND By RESPIRATORY THERAPISTS}

61. Tocino IM, Miller MH, Fairfax WR. Distribution of pneumothorax in the supine and semirecumbent critically ill adult. AJR Am J Roentgenol 1985;144(5):901-905.

62. Ball CG, Kirkpatrick AW, Laupland KB, Fox DL, Litvinchuk S, Dyer DM, et al. Factors related to the failure of radiographic recognition of occult posttraumatic pneumothoraces. Am J Surg 2005; 189(5):541-546.

63. Ding W, Shen Y, Yang J, He X, Zhang M. Diagnosis of pneumothorax by radiography and ultrasonography: a meta-analysis. Chest 2011;140(4):859-866.

64. Brook OR, Beck-Razi N, Abadi S, Filatov J, Ilivitzki A, Litmanovich $\mathrm{D}$, et al. Sonographic detection of pneumothorax by radiology residents as part of extended focused assessment with sonography for trauma. J Ultrasound Med 2009;28(6):749-755.

65. Johnson KMJ, Lehman RE. Acute management of the obstructed endotracheal tube. Respir Care 2012;57(8):1342-1344.

66. Volpicelli G. Sonographic diagnosis of pneumothorax. Intensive Care Med 2011;37(2):224-232.

67. Krishnan S, Kuhl T, Ahmed W, Togashi K, Ueda K. Efficacy of an online education program for ultrasound diagnosis of pneumothorax. Anesthesiology 2013;118(3):715-721.

68. Epstein SK. Extubation. Respir Care 2002;47(4):483-492.

69. Ali ER, Mohamad AM. Diaphragm ultrasound as a new functional and morphological index of outcome, prognosis and discontinuation from mechanical ventilation in critically ill patients and evaluating the possible protective indices against VIDD. Egypt J Chest Dis Tuberculosis 2016;66(2):339-351.

70. Osman AM, Hashim RM. Diaphragmatic and lung ultrasound application as new predictive indices for the weaning process in ICU patients. Egypt J Radiol Nucl Med 2017;48(1):61-66.

71. Peñuelas O, Frutos-Vivar F, Fernández C, Anzueto A, Epstein SK, Apezteguía $\mathrm{C}$, et al. Characteristics and outcomes of ventilated patients according to time to liberation from mechanical ventilation. Am J Respir Crit Care Med 2011;184(4):430-347.

72. Soummer A, Perbet S, Brisson H, Arbelot C, Constantin JM, Lu Q, Rouby JJ; Lung Ultrasound Study Group. Ultrasound assessment of lung aeration loss during a successful weaning trial predicts postextubation distress. Crit Care Med 2012;40(7):2064-2072.

73. Kundra P, Mishra SK, Ramesh A. Ultrasound of the airway. Indian J Anaesth 2011;55(5):456-462.

74. Gupta PK, Gupta K, Dwivedi AN, Jain M. Potential role of ultrasound in anesthesia and intensive care. Anesth Essays Res 2011; 5(1):11-19.

75. Raphael DT, Conard FU 3rd. Ultrasound confirmation of endotracheal tube placement. J Clin Ultrasound 1987;15(7):459-462.

76. Kajekar P, Mendonca C, Gaur V. Role of ultrasound in airway assessment and management. Int J Ultrasound Appl Technol Perioper Care 2010;1(2):97-100.

77. Ezri T, Gewurtz G, Sessler DI, Medalion B, Szmuk P, Hagberg C, et al. Prediction of difficult laryngoscopy in obese patients by ultrasound quantification of anterior neck soft tissue. Anaesthesia 2003;58(11):1111-1114.

78. Shibasaki M, Nakajima Y, Ishii S, Shimizu F, Shime N, Sessler DI. Prediction of pediatric endotracheal tube size by ultrasonography. Anesthesiology 2010;113(4):819-824.

79. Tessaro MO, Salant EP, Arroyo AC, Haines LE, Dickman E. Tracheal rapid ultrasound saline test (T.R.U.S.T.) for confirming correct endotracheal tube depth in children. Resuscitation 2015; 89:8-12.

80. Lakhal K, Delplace X, Cottier JP, Tranquart F, Sauvagnac X, Mercier $\mathrm{C}$, et al. The feasibility of ultrasound to assess subglottic diameter. Anaesth Analg 2007;104(3):611-614.
81. Ding LW, Wang HC, Wu HD, Chang CJ, Yang PC. Laryngeal ultrasound: a useful method in predicting post-extubation stridor. A pilot study. Eur Respir J 2006;27(2):384-389.

82. Song K, Yi J, Liu W, Huang S, Huang Y. Confirmation of laryngeal mask airway placement by ultrasound examination: a pilot study. J Clin Anesth 2016;34:638-646.

83. Chenkin J, McCartney CJ, Jelic T, Romano M, Heslop C, Bandiera G. Defining the learning curve of point-of-care ultrasound for confirming endotracheal tube placement by emergency physicians. Crit Ultrasound J 2015;7(1):14.

84. Abbasi S, Farsi D, Zare MA, Hajimohammadi M, Rezai M, Hafezimoghadam P. Direct ultrasound methods: a confirmatory technique for proper endotracheal intubation in the emergency department. Eur J Emerg Med 2015;22(1):10-16.

85. Das SK, Choupoo NS, Haldar R, Lahkar A. Transtracheal ultrasound for verification of endotracheal tube placement: a systematic review and meta-analysis. Can J Anaesth 2015;62(4):413-423.

86. Thomas VK, Paul C, Rajeev PC, Palatty BU. Reliability of ultrasonography in confirming endotracheal tube placement in an emergency setting. Indian J Crit Care Med 2017;21(5):257-261.

87. Mayo PH, Beaulieu Y, Doelken P, Feller-Kopman D, Harrod C, Kaplan A. American College of Chest Physicians/La Societe de Reanimation de Langue Francaise statement on competence in critical care ultrasonography. Chest 2009;135(4):1050-1060.

88. Cholley BP, Mayo PH, Poelaert J, Vieillard-Baron A, Vignon P, Alhamid S. International expert statement on training standards for critical care ultrasonography. Intensive Care Medicine 2011;37(7): 1077-1083.

89. Heegaard W, Hildebrandt D, Spear D, Chason K, Nelson B, Ho J. Prehospital ultrasound by paramedics: results of field trial. Acad Emerg Med 2010;17(6):624-630.

90. Press GM, Miller SK, Hassan IA, Blankenship R, del Junco D, Camp E, Holcomb JB. Evaluation of a training curriculum for prehospital trauma ultrasound. J Emerg Med 2013;45(6):856-864.

91. Kawooya MG, Goldberg BB, De Groot W, Matovu PD, Malwadde EK, Baltarowich OH. Evaluation of us training for the past 6 years at ECUREI, the World Federation for Ultrasound in Medicine and Biology (WFUMB) Centre of Excellence, Kampala, Uganda. Acad Radiol 2010;17(3):392-398.

92. Stolz LA, Muruganandan KM, Bisanzo MC, Sebikali MJ eat al. Point-of-care ultrasound education for non-physician clinicians in a resource-limited emergency department. Trop Med Int Health 2015; 20(8):1067-1072.

93. Galbois A, Ait-Oufella H, Baudel JL, Kofman T, Bottero J, Viennot $\mathrm{S}$, et al. Pleural ultrasound compared with chest radiographic detection of pneumothorax resolution after drainage. Chest 2010; 138(3):648-655.

94. Bedetti G, Gargani L, Corbisiero A, Frassi F, Poggianti E, Mottola G. Evaluation of ultrasound lung comets by hand-held echocardiography. Cardiovasc Ultrasound 2006;4:34.

95. Chalumeau-Lemoine L, Baudel JL, Das V, Arrivé L, Noblinski B, Guidet B, et al. Results of short-term training of naïve physicians in focused general ultrasonography in an intensive-care unit. Intensive Care Med 2009;35(10):1767-1771.

96. Uppalapati A, Oropello J, Mukkera SR, Jamkhana ZA, DelGiudice R, Sherman B. Efficacy of a training program to achieve competence in lung and pleural ultrasound. Chest 2011;140(4):1033.

97. Mukherjee T, Mehta S, Walker D. The development of an in-house thoracic ultrasound teaching programme on a UK intensive care unit. Intensive Care Med 2011;37:1.

98. UAP Flato, HP Guimarães, G Petisco, F Bezerra, AB Cavalcante, $\mathrm{O}$ Berwanguer. Use of lung ultrasonography in the detection of pneumothorax among medical students and emergency physicians. Crit Care 2011;15(S2):46. 


\section{LUNG UltRASOUND BY RESPIRATORY THERAPISTS}

99. Reissig A, Copetti R, Mathis G, Mempel C, Schuler A, Zechner P. Lung ultrasound in the diagnosis and follow-up of communityacquired pneumonia: a prospective, multicenter, diagnostic accuracy study. Chest 2012;142(4):965-972.

100. Tutino L, Cianchi G, Barbani F, Batacchi S, Cammelli R, Peris A. Time needed to achieve completeness and accuracy in bedside lung ultrasound reporting in intensive care unit. Scand J Trauma Resusc Emerg Med 2010;18:44.

101. Millington SJ, Arntfield RT, Guo RJ, Koenig S, Kory P, Noble V, et al. The Assessment of Competency in Thoracic Sonography (ACTS) scale: validation of a tool for point-of-care ultrasound. Crit Ultrasound J 2017;9:25.

102. Skaarup SH, Laursen CB, Bjerrum AS, Hilberg O. Objective and structured assessment of lung ultrasound competence. A multispecialty Delphi consensus and construct validity study. Ann Am Thorac Soc 2017;14(4):555-60.

103. Mayo PH, Goltz HR, Tafreshi M, Doelken P. Safety of ultrasoundguided thoracentesis in patients receiving mechanical ventilation. Chest 2004;125(3):1059-1062.

104. Poletti PA, Kinkel K, Vermeulen B, Irmay F, Unger PF, Terrier F. Blunt abdominal trauma: should US be used to detect both free fluid and organ injuries? Radiology 2003;227(1):95-103.
105. Ojaghi Haghighi SH, Morteza Begi HR, Sorkhabi R, Tarzamani MK, Zonouz GK, Mikaeilpour A, Rahmani F. Diagnostic accuracy of ultrasound in detection of traumatic lens dislocation. Emergency 2014;2(3):121-124.

106. Heydari F, Esmailian M, Dehghanniri M. Diagnostic accuracy of ultrasonography in the initial evaluation of patients with penetrating chest trauma. Emergency 2014;2(2):81-84.

107. Volpicelli G, Elbarbary M, Blaivas M, Lichtenstein DA, Mathis G, Kirkpatrick AW, et al. International evidence-based recommendations for point-of-care lung ultrasound. Intensive Care Med 2012; 38(4):577-591.

108. Touw HRW, Tuinman PR, Gelissen HPMM, Lust E, Elbers PWG. Lung ultrasound: routine practice for the next generation of internists. Neth J Med 2015;73(3):100-107.

109. Abdalla W, Elgendy M, Abdelaziz AA, Ammar MA. Lung ultrasound versus chest radiography for the diagnosis of pneumothorax in critically ill patients: a prospective, single-blind study. Saudi J Anaesth 2016;10(3):265-269.

110. Koeze J, Nijsten MW, Lansink AO, Droogh JM, Ismael F. Bedside lung ultrasound in the critically ill patient with pulmonary pathology: different diagnoses with comparable chest x-ray opacification. Critical Ultrasound J 2012;4(1):1. 